livraisons

d'Histoire

de l'Architecture

\section{Livraisons de l'histoire de l'architecture}

$21 \mid 2011$

Bâtir et orner

\title{
Orner ou ne pas orner, la série des hommages publics aux Archives nationales
}

Decorating or not, the serie of hommages publics at the Archives nationales Verzieren oder nicht verzieren, die série der öffentlichen Würdigungen in den Archives nationales

\section{Catherine Chevillot}

\section{OpenEdition}

Journals

Édition électronique

URL : http://journals.openedition.org/lha/283

DOI : $10.4000 /$ lha. 283

ISSN : 1960-5994

\section{Éditeur}

Association Livraisons d'histoire de l'architecture - LHA

\section{Édition imprimée}

Date de publication : 10 juin 2011

Pagination : 115-128

ISSN : 1627-4970

\section{Référence électronique}

Catherine Chevillot, "Orner ou ne pas orner, la série des hommages publics aux Archives nationales », Livraisons de l'histoire de l'architecture [En ligne], 21 | 2011, mis en ligne le 10 juin 2013, consulté le 01 mai 2019. URL : http://journals.openedition.org//ha/283 ; DOI : 10.4000/lha.283

Ce document a été généré automatiquement le 1 mai 2019.

Tous droits réservés à l'Association LHA 


\section{Orner ou ne pas orner, la série des hommages publics aux Archives nationales}

Decorating or not, the serie of hommages publics at the Archives nationales Verzieren oder nicht verzieren, die série der öffentlichen Würdigungen in den Archives nationales

\section{Catherine Chevillot}

1 Louis XVIII prit en 1816 un décret soumettant tout hommage public à l'autorisation de l'État: ce sont les archives du bureau chargé du suivi de ces demandes qui feront ici l'objet d'une étude (Archives nationales, sous-série $\mathrm{F}^{1 \mathrm{c}} \mathrm{I}$, Esprit public). Les dossiers concernent majoritairement les changements de noms de rue (environ un tiers), puis les monuments commémoratifs (part variable selon les départements, environ 10 à $20 \%$ ), le reste se répartissant entre concessions gratuites dans les cimetières communaux et quelques rares cas d'hommages personnels (attribution d'une épée d'honneur, buste dans une salle de délibération, etc.). La série se présente matériellement sous la forme de 63 cartons (cotes $\mathrm{F}^{1 \mathrm{c}} \mathrm{I} 137$ à 186, et 189-197 ${ }^{\text {ter }}{ }^{1}$. Pour cette communication, 18 ont été examinés, soit environ un tiers. Le classement est fait par ordre alphabétique de départements, et pour chaque département on trouve systématiquement deux dossiers : un bleu, pour les affaires antérieures à 1870, un blanc pour les affaires postérieures ${ }^{2}$. Des plans, dessins (ill. 1), gravures (ill. 2) et photographies peuvent y être conservés, de façon minoritaire toutefois. 
III. 1 : Projet de monument à Henri Martin

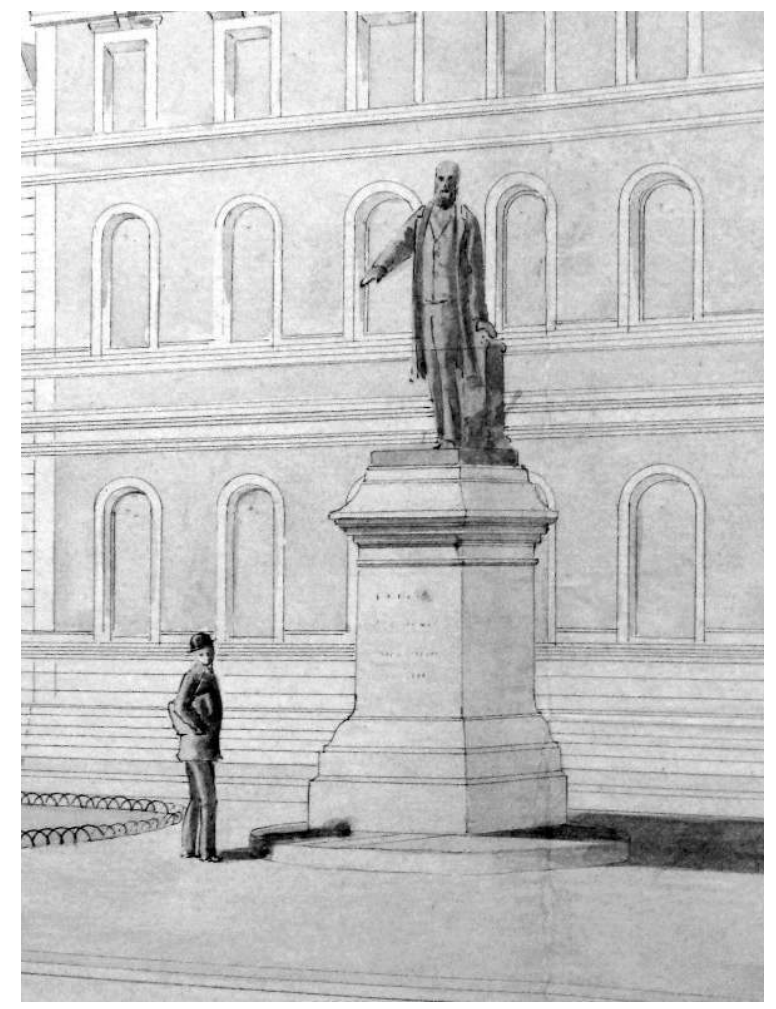

Projet autorisé par décret du 23 mai 1887, Saint-Quentin (Aisne), statuaire : Marquet de Vasselot (dessin de l'architecte). Arch. nat., F/1c(I)/137

CL. DE L'AUTEUR. 
PROJET DU MONUMENT

DU GÉNÉRAL "LE FLO»

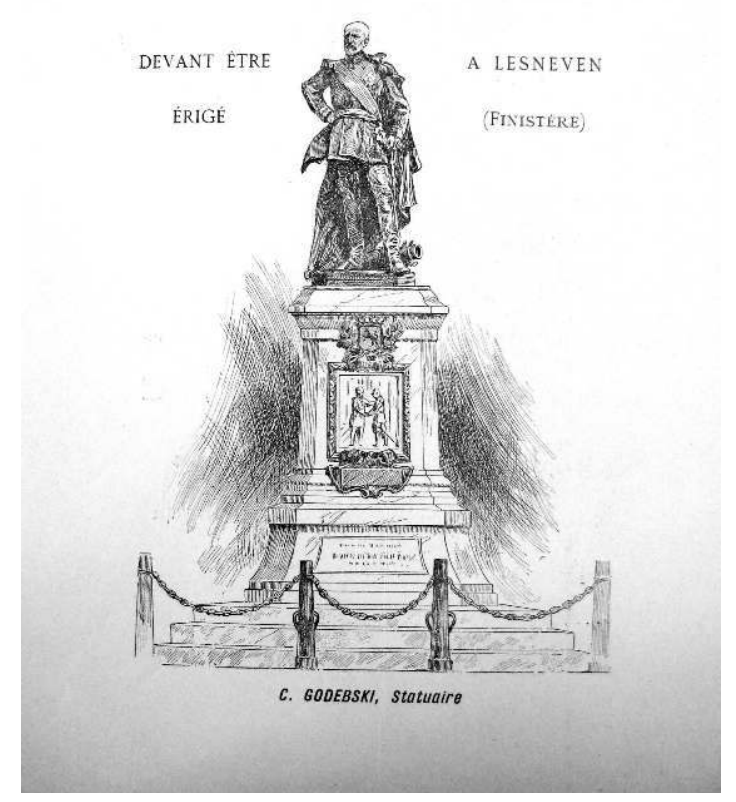

PROJET AUTORISÉ PAR DÉCRET DU 20 AVRIL 1899, LESNEVEN (FINISTÈRE), STATUAIRE, CYPRIEN GODEBSKI (PUBLICITÉ). ARCH. NAT., F/1C(I)/147

CL. DE L'AUTEUR.

2 Le contenu des dossiers s'explique par le fonctionnement administratif du traitement administratif : les préfets adressent au ministère les projets de monuments, le service les traite souvent en lien étroit avec le ministre d'État (sous le Second Empire) et le cabinet du ministre de l'intérieur. Il peut arriver que l'on demande son avis au service des bâtiments civils, ou à d'autres ministères : ministère de l'instruction publique et des beaux-arts, ministère de la guerre (pour les hommages aux militaires), ministère de la justice (pour les magistrats).

3 À dire vrai, les cas de censure avérée et militante sont rares; les dossiers permettent en revanche de percevoir les orientations et les influences qui affectent le mouvement général de la statuaire commémorative. En nous concentrant sur la période antérieure à 1870, et particulièrement sur le Second Empire ${ }^{3}$, nous nous attacherons à observer le mécanisme de ce contrôle. On note que seuls les projets qui émanent d'une instance collective connaissent une suite (ill. 3). Les initiatives strictement individuelles sont écartées, même lorsqu'elles sont favorables au régime : ainsi une veuve, protestant de sa sympathie pour l'Empire, et voulant offrir un monument à Napoléon III à sa commune (Saint-Christoly, Gironde, 1858, $\mathrm{F}^{1 \mathrm{c}} \mathrm{I} 149$; ill. 4), ne parvint jamais à ses fins. 
III. 3 : pétition en faveur d'un monument à Napoléon ler, à Fouras, près de Rochefort (CharenteInférieure)

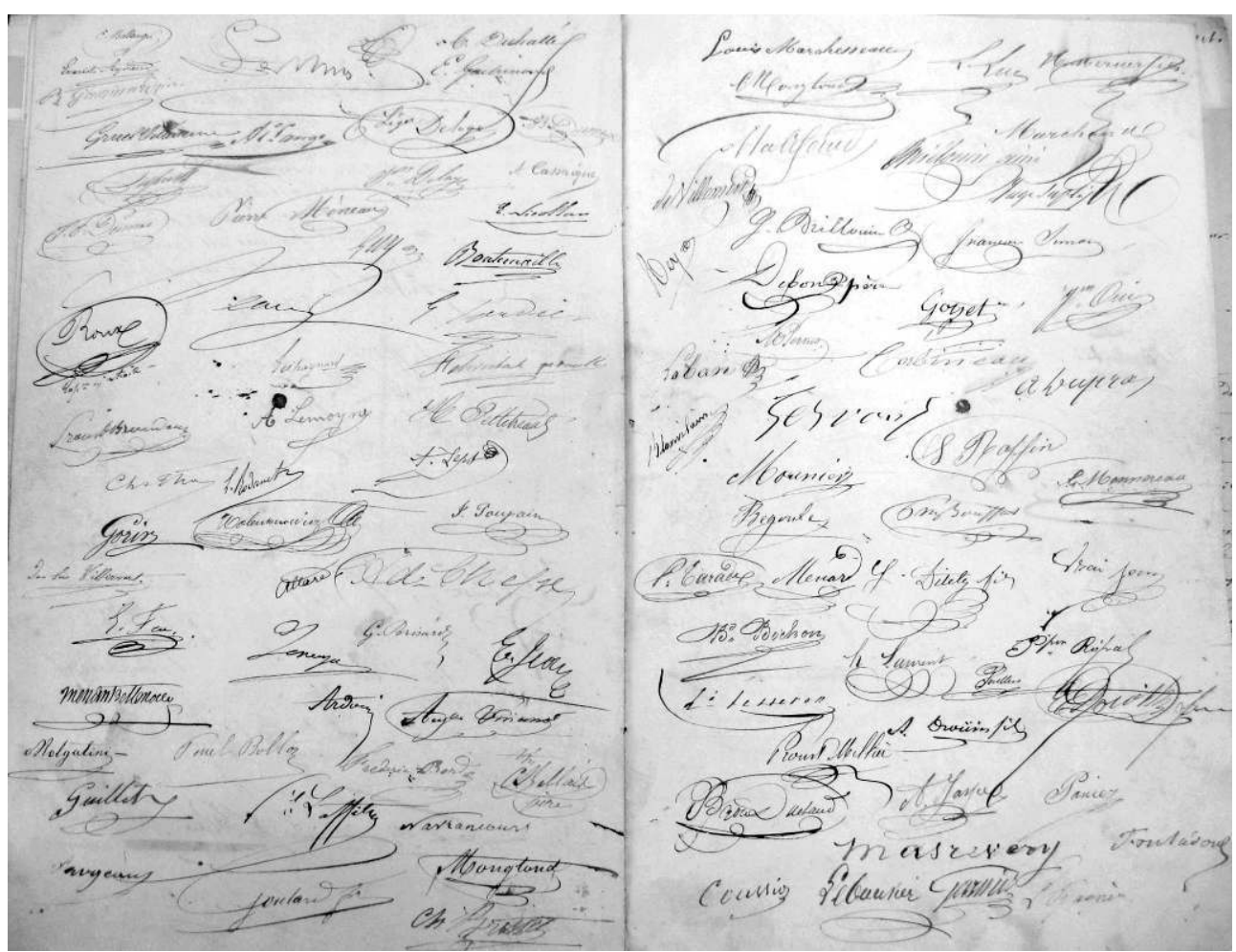

PROJET NON AUTORISÉ. ARCH. NAT., F/1C/I/142

CL. DE L'AUTEUR 
III. 4 : Annotation du ministre sur le rapport de son cabinet concernant un projet de monument à Napoléon III, à Saint-Christoly (Gironde)

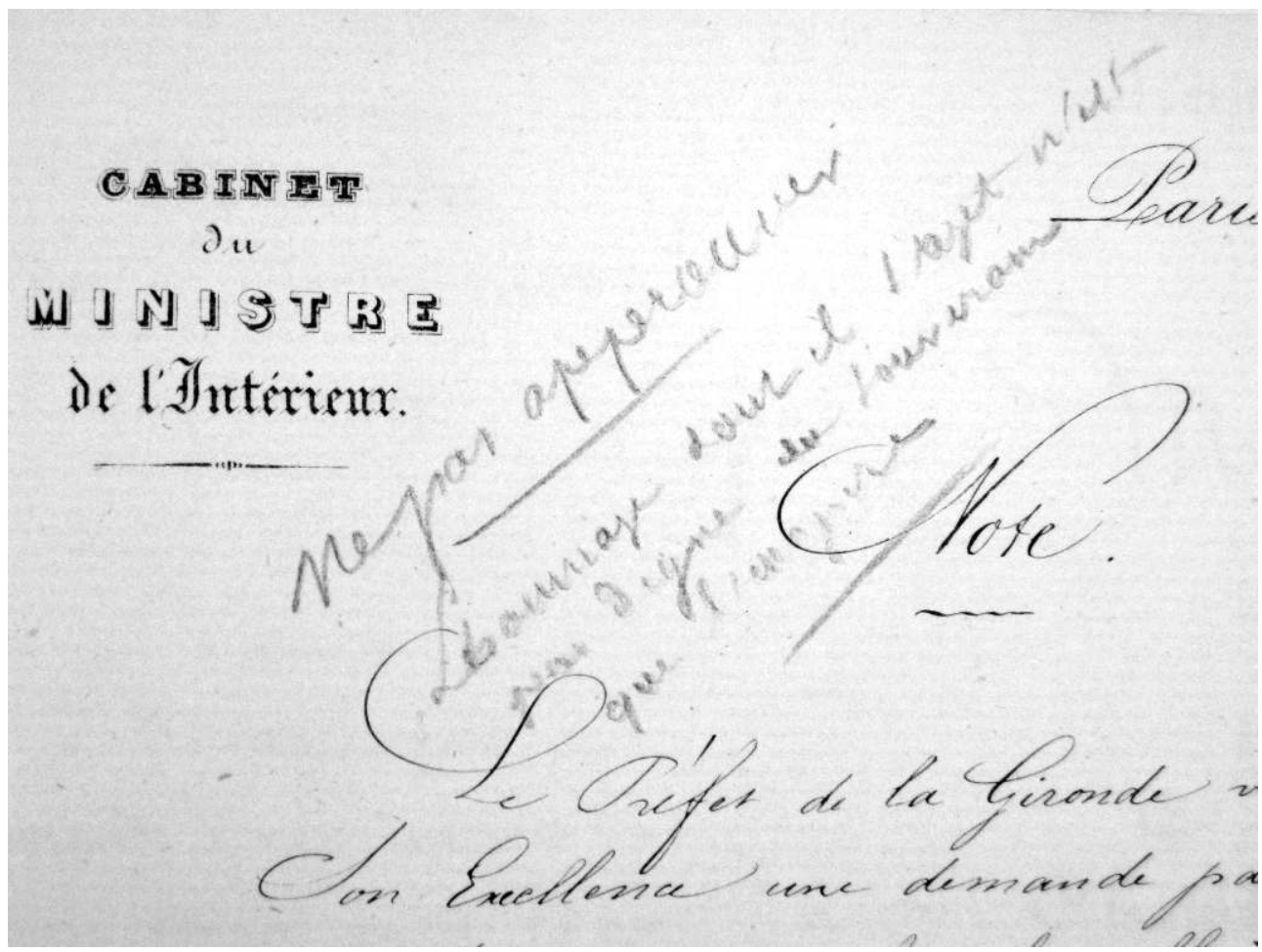

PROJET NON AUTORISÉ. ARCH. NAT., F/1C(I)/149

CL. DE L'AUTEUR.

\section{Les avis délibérément négatifs pour motifs politiques}

4 On trouve bien néanmoins des cas de refus pour des motifs politiques. En 1858, le préfet de l'Ain informe que Sudour, un ancien soldat de l'armée napoléonienne résidant à Genève, intervient de manière répétée via le consul de France à Genève pour faire ériger un Monument à Napoléon Ier à Ferney (Ain, $\mathrm{F}^{1 \mathrm{c}}$ I 137). Cette affaire, indique le Consul, « nous créerait ici des ennuis si on donnait suite à ses idées » et le préfet signale au ministre que « d'après la connaissance personnelle que j'ai de la situation sur la frontière du pays de Gex, l'opinion de M. le Consul est la mienne ». Il organise la riposte « afin de le prémunir contre les écarts de la presse genevoise si le prospectus [...] venant à tomber entre ses mains, elle en induisait [...] l'approbation du gouvernement français aux projets de M. Sudour " (lettre du 23 septembre 1858). En effet, les relations entre France et Suisse sont alors tendues à l'extrême. On sort à peine de l'affaire de Neuchâtel (1856-1857), crise suscitée par la volonté de la Suisse d'obtenir un débouché sur le Rhin. Chose rare, le pays avait même mobilisé ses troupes et abritait les réfugiés de Bourbaki. En 1860, on allait connaître une nouvelle crise, dite "affaire de Savoie", déclenchée par les ambitions rivales des deux pays, la Suisse revendiquant une partie de la Savoie pour y établir un canton. On comprend donc que le projet du soldat Sudour, qui pourtant se prévalait de représenter de nombreux soldats du premier empereur, n'ait pas suscité l'enthousiasme.

La commune de Murgron (Landes, $\mathrm{F}^{1 \mathrm{C}} \mathrm{I}$ 152) voulut en 1869 ériger un Monument à Frédéric Bastiat, économiste qui fut longtemps juge de paix à Mugron, mais aussi ancien 
représentant à la Constituante. Le préfet informe en ces termes sa tutelle : «Cette pensée, M. le Ministre, n'est provoquée par aucun sentiment hostile, et ce n'est pas à l'homme politique, à l'ancien député de la République que l'on entend ainsi rendre hommage mais à l'économiste. Toutefois, au moment où va s'ouvrir la campagne électorale, il pourrait $\mathrm{y}$ avoir de graves inconvénients à ce que ce projet fût mis en relief. Les gens hostiles, les journaux d'opposition, dénaturant le mobile véritable de ses auteurs ne manqueront sans doute pas de profiter de cette circonstance pour proclamer que l'œuvre est avant tout un témoignage à la mémoire de l'homme de 1848 , et de représenter comme un acte d'opposition une manifestation qui se baserait sur un tout autre sentiment ». Le cabinet du ministre écrit en marge: "Grande réserve, ne pas s'engager ». Le maire saura «obtenir que le projet soit ajourné jusqu'après les élections » (27 mars 1869). Le décret fut finalement pris par crainte que le refus ne provoque encore plus de réactions.

6 L'administration montre une capacité étonnante à triompher des plus longs acharnements : pour réclamer un Monument au général Dampierre (Troyes, 1859, $\mathrm{F}^{1 \mathrm{c}} \mathrm{I} 139$ ), le fils de l'officier harcèle véritablement tout le monde à l'aide de lettres fleuve depuis 1836. Hier comme aujourd'hui, l'inertie s'avère payante, aucun décret n'est pris, et Troyes n'eut jamais de monument à Dampierre ${ }^{4}$. Bien évidemment, le régime impérial ne devait avoir aucune envie d'élever un monument à un officier qui avait fait toute sa carrière durant la Révolution, et était mort au combat en 1793, sans avoir été sous le commandement de Napoléon I ${ }^{\mathrm{er}}$.

7 De manière générale, les archives mettent surtout en évidence le succès du phénomène d'auto-censure, ou plus exactement de zèle pro-gouvernemental. Dans les années qui suivent le coup d'État, on voit fleurir les projets de monuments à des maréchaux d'Empire (Serrurier, Laon, 1862 ; Baron Ladoucette, Gap, 1861) ou à des dignitaires du régime (duc De Cazes, Decazevilles, 1860 ; Montalivet, Valence, 1867), à Napoléon $\mathrm{I}^{\mathrm{er}}$, ainsi que des projets à l'évidence flagorneurs ou serviles: monument au Prince impérial, ou à l'Impératrice Eugénie (Toulouse, 1854), ces deux derniers d'ailleurs sans suite.

8 Il n'y a pratiquement pas de demande ouvertement anti-impériale. Il est des cas à propos desquels on pourrait se poser la question, mais il semble qu'elles soient davantage dues à une maladresse pataude qu'à une volonté de provocation. Citons deux exemples. La commune corse de Bastelica adresse une pétition à l'Empereur pour élever un monument à Sampierro Corso $\left(\mathrm{F}^{1 \mathrm{c}} \mathrm{I} 143 ; \text { ill. } 5\right)^{5}$, qui commence par cette phrase délicieuse : « Si la providence n'avait fait naître en Corse l'auguste famille Bonaparte, Sampierro eût été le

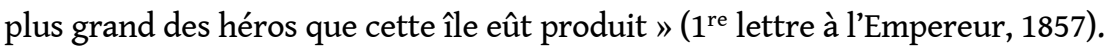




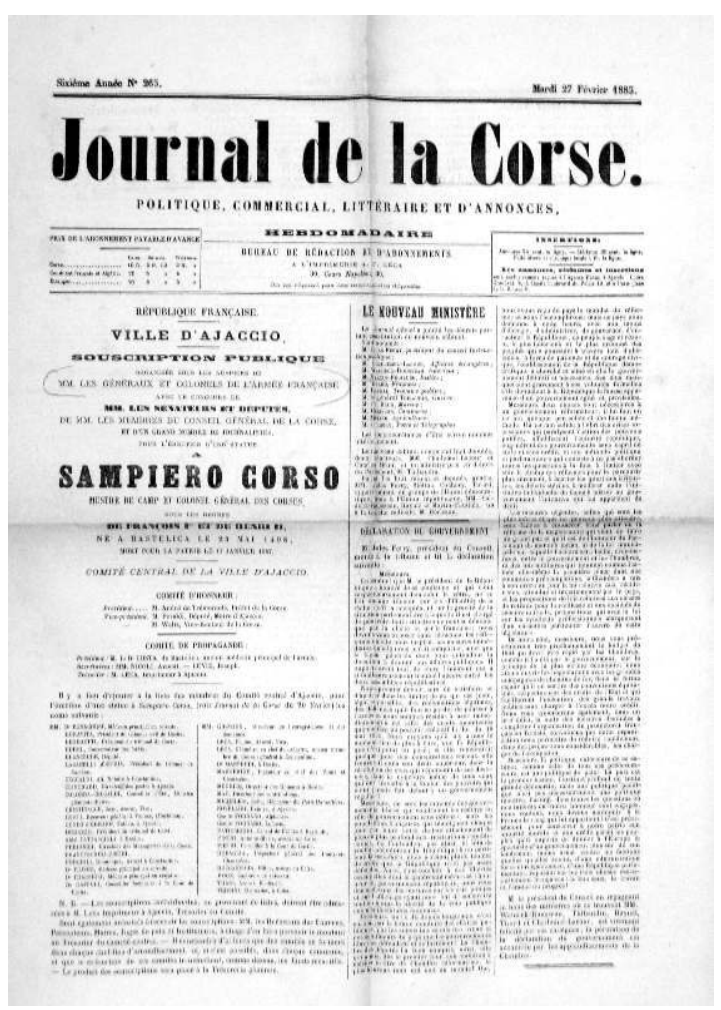

PROJet AUTORISÉ PAR DÉCRET dU 12 SEPTEMBRE 1890. ARCH. NAT., F/1C(I)/143

CL. DE L'AUTEUR.

On comprend assez bien qu'elle n'ait pas incliné l'autorité bonapartiste à honorer un héros concurrent du sien! Le dossier fut clos après une mention manuscrite en marge, qualifiant le projet de " pas opportun ». Il ne ressurgit qu'en 1883, après l'affermissement de la République: "il [le projet] me semble être, dans l'intérêt bien compris du gouvernement de la République, d'accéder aux désirs de la population», peut-on lire alors. "Pendant l'Empire, en effet, tout ce qui paraissait rappeler les libertés perdues était mal accueilli par les hommes au pouvoir, et d'un autre côté les sollicitations exclusivement personnelles dont on fatiguait les courtisans de Napoléon III primaient l'intérêt général. [...] Il fut, et nous devons surtout le considérer à ce point de vue, le premier Corse qui mit son épée au service de la France, qu'il aimait à l'égal de sa Patrie.» Les partisans du monument ont formé une « réunion où se trouvaient les représentants les plus notables de toutes les opinions politiques: républicains, bonapartistes, légitimistes, orléanistes. Je pense qu'il est d'une habile administration et d'une sage tactique gouvernementale de saisir cette occasion pour faire oublier, autant que possible, l'acuité et la violence des luttes politiques antérieures » (lettre du préfet, 17 mars 1883). Le ministre de l'intérieur est alors Waldeck-Rousseau. Le monument fut inauguré 21 septembre 1890.

10 Le cas de La Boëtie (Sarlat, Dordogne, $\mathrm{F}^{1 \mathrm{c}} \mathrm{I}$ 145), en revanche, est sans doute délibérément militant. Refusé en 1861, le monument est autorisé en 1890 : La Boëtie, « en plein siècle de la servitude et du despotisme monarchique, fut le premier revendicateur de la souveraineté nationale »: voilà un programme clair. Le conseil municipal souligne que 
«La Boëtie a été en France le premier apôtre de la liberté, de l'égalité et de la fraternité, et que par son magnifique pamphlet il a puissamment contribué à l'enfantement de nos libertés et à l'essor de la civilisation » (délibération du 27 avril 1876 ; finalement confié à Tony-Noël en 1890).

\section{Les acteurs extérieurs et les pressions}

11 Mais le fonds est aussi le moyen de comprendre les divers forces en présence et la manière dont elles influent sur le succès ou l'échec des initiatives. Dans les cas où le projet rencontre un intérêt ou un appui particulier de l'autorité centrale, comme par le Monument à Berryer, Marseille (1870, par Barre, $\left.\mathrm{F}^{1 \mathrm{c}} \mathrm{I} 140\right)$, l'aboutissement est diligent, comme le montre la lettre du ministre au préfet des Bouches-du-Rhône : "Je tiens à vous dire confidentiellement que le projet d'érection d'une statue de M. Berryer a été accueilli favorablement par le gouvernement [...] Sans intervenir dans cette affaire, vous ferez toutefois bien de vous y montrer personnellement favorable » (5 mars 1870).

Il arrive que l'Empereur marque un intérêt personnel pour tel ou tel projet, en ajoutant, au moment où on lui présente les projets de décret, une contribution sur le budget de sa maison: Monument à la bataille de Montmirail, 1814 (1864, Montmirail, Aisne, F ${ }^{1 \mathrm{c}}$ I 137), auquel il souscrit pour 4000 francs. (ill. 6 et 7).

\section{6 : Projet pour le monument à la bataille de Montmirail (Aisne)}

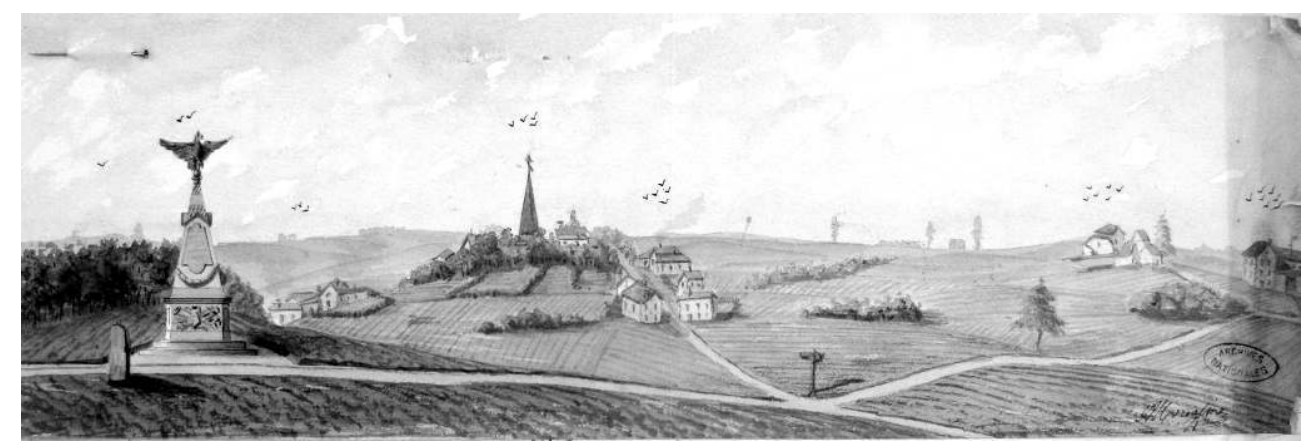

PROJet AUTORISÉ PAR DÉCret en 1864 (DESSIN AQUARELLÉ). ARCH. NAT., F/1C(I)/137

CL. DE L'AUTEUR. 
III. 7 : Projet pour le monument à la bataille de Montmirail (Aisne)

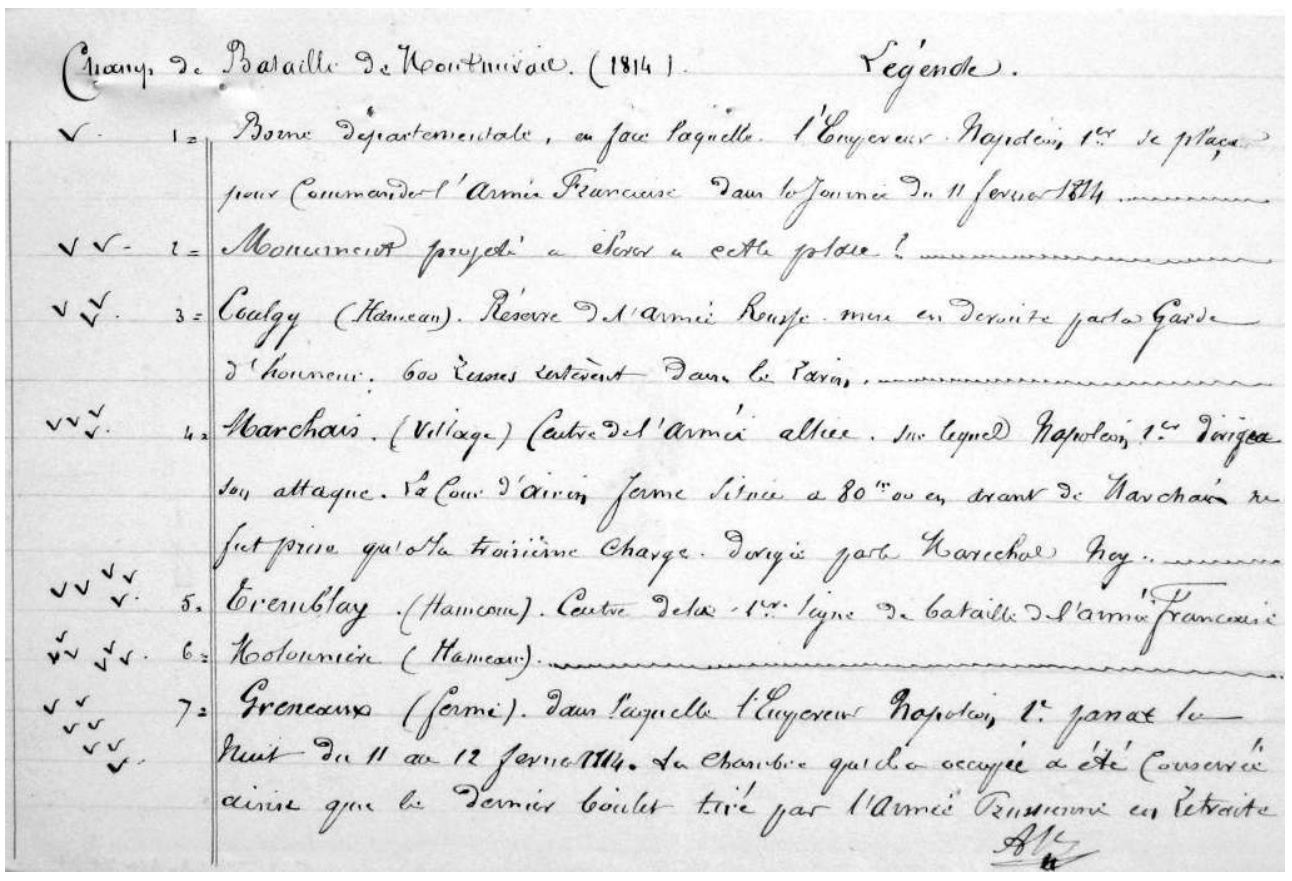

LÉgENDE ORIgINALE DU DESSIN AQUARELLÉ. ARCH. NAT., F/1C(I)/137

CL. DE L'AUTEUR.

13 C'est le cas pour la Vierge du Puy (pour laquelle on ne s'attendrait pas a priori à un dossier d'autorisation pour un hommage public...) : l'Empereur ayant accordé 15000 francs à la souscription, le ministère de l'intérieur suivit très attentivement le chantier, $\mathrm{y}$ compris les plans techniques et les rapports de l'architecte Gisors.

Il arrive même que le corps préfectoral, évidemment non sans visée carriériste, soit luimême à l'initiative de sculptures favorisant la propagande du régime. Le préfet de l'Isère s'adresse ainsi au ministre en 1861: «À mon arrivée dans ce département dont les sentiments napoléoniens m'étaient depuis longtemps connus, j'ai été surpris, je l'avoue, de n'y trouver aucun monument qui rappela une tradition si populaire [la scène de Laffrey au retour de l'Ile d'Elbe en 1815], et depuis lors, j'avais songé plus d'une fois aux moyens de combler cette lacune " ( $\left.\mathrm{F}^{1 \mathrm{c}} \mathrm{I} 152\right)$. Irvoy vient lui présenter un projet dont il souligne la portée idéologique, «facilement accessible aux masses ». Il propose au conseil général de verser 20000 francs pour le monument «dont l'initiative doit vous appartenir » (approuvé le 30 août 1861). Le résultat ne se fait pas attendre : "J'ai soumis cette affaire à l'Empereur et je m'empresse de vous informer [...] que S. M. désire que le monument dont il s'agit soit élevé à Grenoble et non à Laffrey » (lettre du ministre d'État, 17 avril 1862). Le Monument à Napoléon $I^{e r}$, d'abord confié à Barye puis à Fremiet, fut inauguré le 17 août 1868, place de Verdun, devant la préfecture, mais déboulonné dès 1870. Il fut restauré et mis en place au bord du lac de Laffrey au XX ${ }^{\mathrm{e}}$ siècle.

Les évêques peuvent également intervenir dans les procédures, plus rarement mais alors de façon très forte: en 1857, le maire de Saint-Martin-Lys (Aude, $\mathrm{F}^{1 \mathrm{c}} \mathrm{I} 139$; ill. 8 et 9) expose le désir de la commune de rendre hommage à l'abbé Félix Armand (statue par Bonnassieux), créateur de la route entre Quillan et Roquefort, percée dans les gorges de l'Aude pour désenclaver Saint-Martin. L'abbé « qui a sacrifié sa carrière à une tâche 
pénible et obscure » avait commencé seul la percée au lieu dit «le trou du curé », puis dirigé les travaux des habitants en 1780 (lettre du maire, 27 mars 1857).

III. 8 : Plan des gorges de Quillan à Saint-Martin-Lys (Aude), en vue de l'implantation du monument à l'abbé Félix Armand

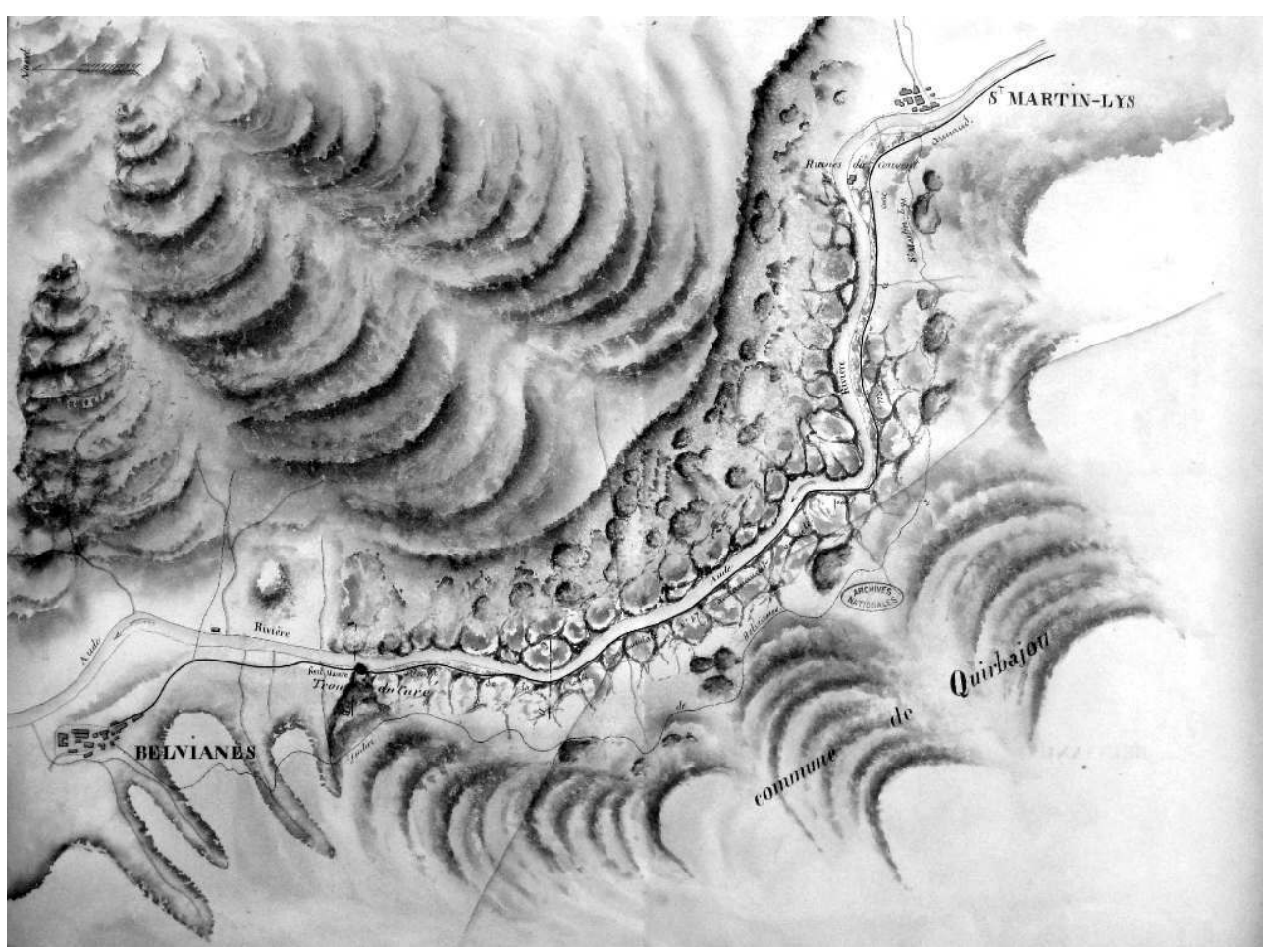

PROJET AUTORISÉ PAR DÉCRET DU 29 SEPTEMBRE 1859. ARCH. NAT., F/1C(I)/139

CL. DE L'AUTEUR. 
III. 9 : Coupe des gorges de Quillan à Saint-Martin-Lys (Aude), en vue de l'implantation du monument à l'abbé Félix Armand

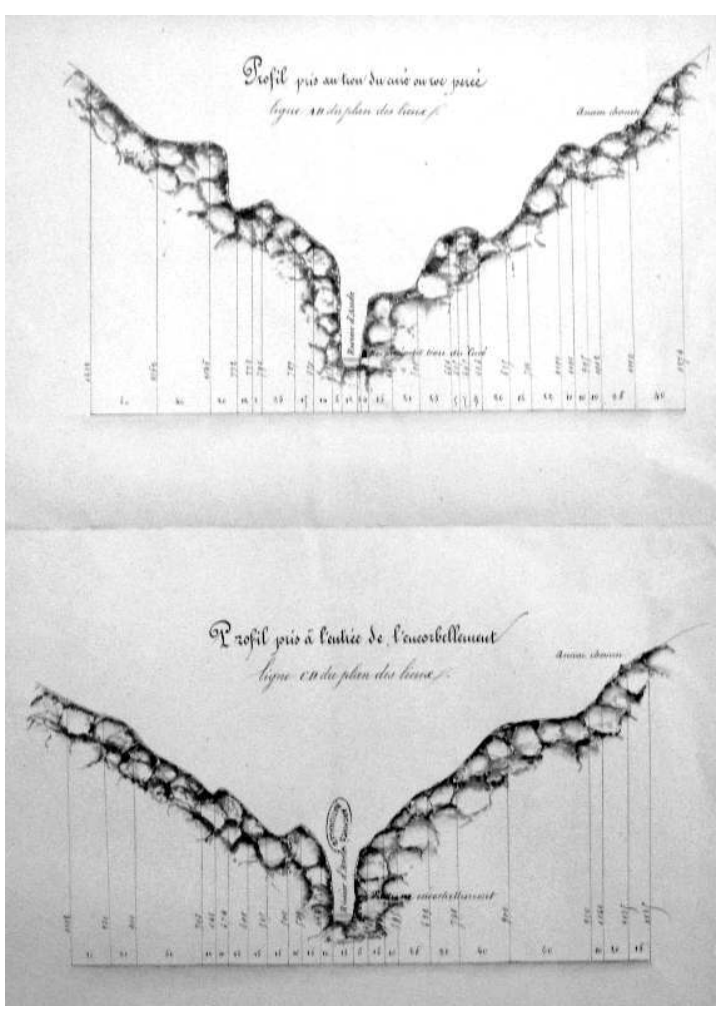

ARCH. NAT., F/1C(I)/139

CL. DE L'AUTEUR

Le préfet de l'Aude institue une commission directement placée sous son autorité, qui organise la réunion des fonds, les souscriptions, etc., présidée par l'évêque de Carcassonne, et réunissant maires et curés de Quillan (commune où aboutit la route) et de Saint-Martin. Un décret du 17 octobre 1857 prescrit que « la commune de Saint-MartinLys est autorisée à ériger un monument ». Mais l'évêque intervient, trouvant que la statue “ne peut être élevé[e] dans un petit village ;j'en avais entretenu le duc de Padoue ; un des frères de l'évêque est sous-préfet de Verdun et doit sa carrière à son Excellence" (15 décembre 1859). Le curé de Saint-Martin a beau réclamer justice : "Sire, successeur du curé Armand, je croirais manquer à ce que je dois à sa mémoire et à ses paroissiens en ne protestant pas par tous les moyens contre la décision de Monseigneur prise sans nous consulter, nous, les principaux intéressés, décision que j'attribue moins à la mauvaise volonté de Monseigneur, mon supérieur qu'aux obsessions et aux prévenances dont sont circonvenus des citadins puissants. ». Rien n'y fait, et le décret est modifié le 28 décembre 1859, autorisant l'érection à Quillan. 


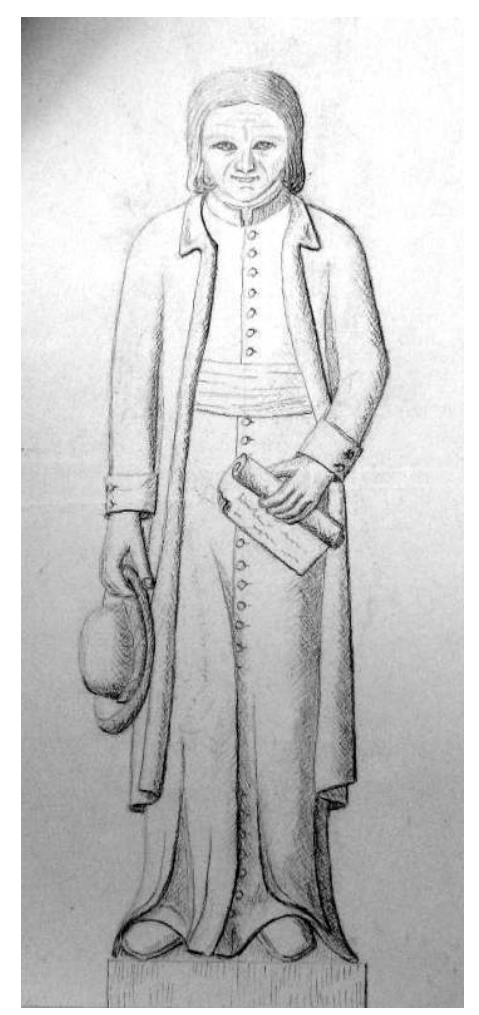

PROJET AUTORISÉ PAR DÉCRET dU 24 SEPTEMBRE 1876 (DESSIN AU TRAIT). ARCH. NAT., F/1C(I)/145 CL. DE L'AUTEUR.

Mais le ton change après 1875 : la commune d'Arc-sous-Montenot (Doubs, $\mathrm{F}^{1 \mathrm{c}} \mathrm{I} 145$; ill. 10) souhaite élever un monument à l'abbé Maillard (1759-1823), conciliateur de procès, sauveur de vies sous la Révolution, conseiller pour les paysans en matière d'agriculture... L'évêque est en désaccord avec l'emplacement proposé : «J'ajouterais que l'emplacement dont il s'agit est tellement à proximité de l'église qu'il en est en quelque sorte une dépendance et que d'après les règles, on ne saurait y placer la statue d'un personnage, quelque respectable qu'il soit, mais que l'Église n'honore pas d'un culte public » (lettre de l'évêque, 7 avril 1876). Cette fois, la commune proteste et obtient gain de cause. Le décret est signé le 24 septembre 1876.

Enfin, l'autorité militaire est particulièrement sourcilleuse quant aux distinctions accordées, en particulier à de simples soldats : l'artilleur Triaire, auteur d'un fait d'armes héroïque (il avait en l'an VIII fait sauter un fort en Algérie pour décimer l'armée musulmane qui y entrait) était originaire du Vigan (1862, Gard, $F^{1 c}$ I 147). Mais la commune ne parvient pas à obtenir l'autorisation car le ministère de la guerre ne retrouve pas la preuve de l'identité du kamikaze de l'armée révolutionnaire. Le même ministère s'opposa un certain temps à l'érection d'un monument au général Travot à Poligny (1861-1863, Jura, $\mathrm{F}^{1 \mathrm{c}} \mathrm{I}$ 152), car celui-ci avait déjà un monument dans une autre ville.

Bien sûr, les députés interviennent de temps à autre, moins toutefois qu'on ne pourrait le penser. Ils écrivent au ministère surtout lorsqu'un dossier s'enlise (Rouget de L'Isle, Lonsle-Saunier), et leur lettre est alors en général assez efficace. Dans la grande majorité des cas, l'État joue - comme aujourd'hui du reste à travers les commissions diverses - un rôle 
de régulateur (y a-t-il des moyens pour financer le projet?) ou d'arbitre des conflits locaux lors de la mise en place de certains projets. On chercha ainsi longtemps un emplacement à Soissons pour installer un Monument à Paillet, avocat à la cour impériale (1857, par Duret, $\mathrm{F}^{1 \mathrm{c}} \mathrm{I}$ 137). Le préfet demande que l'on cherche une autre solution que la place de l'hôtel de ville, trop officielle. Le maire propose la cour du palais de justice. Mais « M. le ministre de la justice, consulté, a exprimé la pensée que l'enceinte d'un palais de justice devait être exclusivement réservée pour les honneurs à rendre à des magistrats " (lettre du maire, 25 octobre 1858). Le décret est signé au bout de trois ans de tergiversation, le 13 février 1861. De même le monument à Monseigneur Affre (Rodez, par Jean-Auguste Barre) attend durant 10 ans le décret, installé sur un socle provisoire qui se délabre (décret du 2 septembre 1873).

Après le Second Empire, on constate que plus on avance dans la Troisième République, moins le fonds compte de demandes d'autorisation, surtout si l'on songe qu'au contraire, le nombre de statues et bustes commémoratifs entre dans l'ère de la "statuomanie ". Peut-être le fonds est-il lacunaire, mais on a bien l'impression que l'usage se perd, car les fonctionnaires du bureau relèvent les traces d'inauguration dans la presse, pour lesquels aucune demande n'a été déposée. Plus on va vers 1900, plus le nombre de décrets devient ridicule au regard du nombre de monuments effectivement érigés.

Le fonds des hommages publics doit bien sûr être croisé avec d'autres sources. Un dossier correspondant se trouve, de manière très logique, dans les archives départementales. Les plus gros dossiers permettant de suivre tous les aspects de l'histoire du monument se trouvent dans les archives municipales (série $\mathrm{M}$, sauf lorsque les archives, pour le plus grand désespoir du chercheur, ont été recotées en W). En effet, dans le fonds $\mathrm{F}^{1 \mathrm{c}} \mathrm{I}$, bien des aspects sont passés sous silence : le nom du sculpteur n'apparaît quasiment jamais, la dimension esthétique n'est pas abordée. Enfin le cédérom À nos grands hommes, la sculpture publique de la Révolution à la seconde guerre mondiale (musée d'Orsay - institut national de l'histoire de l'art - France Debuisson / Laurent Chastel, Paris, 2004) présente un état de la recherche sur environ 5000 monuments, incluant déjà partiellement le résultat d'un premier dépouillement du fonds des hommages publics.

\section{NOTES}

1. . Je tiens à remercier particulièrement Denise Ogilvie, responsable de la série $\mathrm{F}^{1 \mathrm{c}}$, qui m'a donné accès au fonds dans des conditions privilégiées, ainsi que Catherine Mérot et Nadine Gastaldi.

2. . En fait, le classement n'est pas aussi rigoureux, et les dossiers bleus contiennent souvent des dossiers postérieurs à 1870 , mais il n'est pas exclu que cela ait un sens.

3. Certains cartons conservent des dossiers de la monarchie de Juillet (surtout la Seine), mais cela est peut fréquent. Ce n'est pour autant pas étonnant : la fréquence des demandes suit assez bien, semble-t-il le rythme de développement du phénomène du monument public, qui ne commence vraiment à prendre de l'ampleur qu'avec le Second Empire.

4. . Mais le fils du général avait eu plus de succès avec Louis-Philippe, puisqu'une colonne avait été érigée à Valenciennes en 1836. 
5. Ce guerrier du XVII ${ }^{\mathrm{e}}$ siècle a combattu avec la France pour l'indépendance de son pays au XVI e siècle, contre les Génois.

\section{RÉSUMÉS}

Louis XVIII prit en 1816 un décret soumettant tout hommage public à l'autorisation de l'état : les archives du bureau chargé du suivi de ces demandes font ici l'objet d'une étude globale en ce qui concernent les monuments commémoratifs (Arch. nat., sous-série $\mathrm{F}^{1 \mathrm{c}} \mathrm{I}$ ). La recherche montre que les cas de censure avérée et militante sont rares, mais les dossiers permettent en revanche de percevoir les orientations et les influences qui affectent le mouvement général de la statuaire commémorative. En concentrant le propos sur la période antérieure à 1870, et particulièrement sur le Second Empire, on observer le mécanisme de ce contrôle. Après le Second Empire, on constate que, plus on avance dans la Troisième République, plus le nombre de décrets devient ridicule au regard du nombre de monuments effectivement érigés: le contrôle, s'il reste légalement obligatoire, devient dans la réalité très épisodique.

Louis XVIII issued in 1816 a decree subjecting each and every public homage to the authorization of the State: this article is a global study of archives regarding the applications for memorials made by the office in charge of following up on these requests (Archives Nationales, sub-series $\mathrm{F}^{1 \mathrm{c}}$ I). It shows that cases of established and militant censure are rare. But these files also give us an insight on orientations and influences governing overall the movement of commemorative statuary. By focusing on the period prior to 1870, particularly on the Second Empire, it becomes possible to discern the mechanisms of this control. After the Second Empire, one can notice that the more the French Third Republic goes on, the more the number of decrees shrinks dramatically compared to the number of monuments actually built: if the control is still legally mandatory, in reality it becomes only occasional.

Ludwig XVIII. gab 1816 einen Erlass heraus, der besagt, dass jede öffentliche Würdigung erst vom Staat genehmigt werden sollte : es handelt sich hier um die Darstellung der Archive des für die Genehmigungsanträge zuständigen Dienstes, insbesondere die in ihrer Gesamtheit durchsuchte sous-série $F^{1 c} I$, die die Gedenkstätten betrifft. Wenn auch erwiesenermaßen scharfe Zensurfälle ganz selten vorkommen, bieten diese Unterlagen reichhaltige Informationen über die Umwandlungen und die Einflüsse im Bereich der Bauplastik der Gedenkstätten. Der Inhalt dieses Berichts konzentriert sich auf die Zeit vor 1870, besonders auf das Second Empire, wo die Organisation dieser Kontrolle am besten vor Augen gestellt wird. Nach dem Second Empire ergibt es sich, dass im Laufe der III. Republik die Menge der Erlasse immer weniger wird im Vergleich zu der wachsenden Menge der tatsächlich errichteten Gedenkstätten : die Kontrolle, die jedoch noch rechtsverbindlich bleibt, wird in der Praxis immer unregelmäßiger.

\section{AUTEUR}

\section{CATHERINE CHEVILLOT}

Catherine Chevillot, de formation universitaire et conservateur depuis 1987, a été conservateur au musée de Grenoble (1988), au musée d'Orsay (1990) et au centre de recherche et de 
restauration des musées de France (1999). Elle est depuis 2003 chef du service de la recherche du musée d'Orsay et a été nommée à la section Sculpture en juin 2008. Parmi ses travaux, figurent les expositions sur les sculpteurs Emmanuel Frémiet, Denys Puech, François Pompon, et, en collaboration, Auguste Préault. Elle a publié le Catalogue des collections du XIXe siècle du musée de Grenoble, et assuré avec Chantal Georgel la direction de À nos grands hommes, répertoire de 5000 monuments sculptés. Elle poursuit des travaux sur l'histoire des techniques et les Salons au XIX siècle. Elle prépare le catalogue des collections de sculpture du musée des Beaux-Arts de Lyon et a été le commissaire de l'exposition sur la sculpture à Paris entre 1905-1914 : Oublier Rodin (9 mars - 30 mai 2009). 